\title{
Controle do Câncer de Mama - Documento de Consenso
}

\author{
Consensus for the Management of Breast Cancer
}

\section{INTRODUÇÃO}

A O rganização Mundial da Saúde estima que, por ano, ocorram mais de 1.050 .000 casos novos de câncer de mama em todo o mundo, o que o torna o câncer mais comum entre as mulheres. No Brasil, não tem sido diferente. Informações processadas pelos Registros de Câncer de Base Populacional, disponíveis para 16 cidades brasileiras, mostram que na década de 90 este foi o câncer mais freqüente no país. As maiores taxas de incidência foram observadas em São Paulo, no Distrito Federal e em Porto Alegre.

Além disso, o câncer de mama constitui-se na primeira causa de morte por câncer entre as mulheres, registrando-se uma variação percentual relativa de mais de $80 \%$ em pouco mais de duas décadas: a taxa de mortalidade padronizada por idade, por 100.000 mulheres, aumentou de 5,77 em 1979, para 9,74 em 2000 (M inistério da Saúde, 2002).

Internacionalmente, tem-se observado em alguns países desenvolvidos, como é o caso dos Estados U nidos, C anadá, Reino U nido, H olanda, D inamarca e N oruega, um aumento da incidência do câncer de mama acompanhado de uma redução da mortalidade por esse câncer, o que está associado à detecção precoce por meio da introdução da mamografia para rastreamento e à oferta de tratamento adequado. Em outros países, como no caso do Brasil, o aumento da incidência tem sido acompanhado do aumento da mortalidade, o que pode ser atribuído, principalmente, a um retardamento do diagnóstico e da instituição de terapêutica adequada.

Frente às limitações práticas para a implementação junto à população de estratégias efetivas para a prevenção do câncer de mama, as intervenções, do ponto de vista da Saúde Pública, passam a ser direcionadas a sua detecção precoce, com a garantia de recursos diagnósticos adequados e tratamento oportuno.

O M inistério da Saúde, considerando a situação atual do câncer de mama no Brasil e percebendo a necessidade de definir as estratégias a serem priorizadas para o seu controle a partir de um trabalho conjunto entre 0 Instituto $\mathrm{N}$ acional de Câncer e a Área Técnica da Saúde da M ulher, com o apoio da Sociedade Brasileira de $M$ astologia, realizou, nos dias 13 e 14 de novembro de 2003, uma oficina de trabalho para discussão e aprovação de recomendações referentes ao controle do câncer de mama. Participaram, além de técnicos de diferentes áreas do $M$ inistério, gestores, pesquisadores que atuam na área de controle de câncer e representantes de Sociedades Científicas afins e de entidades de defesa dos direitos da mulher.

Este documento de consenso apresenta as recomendações emanadas dessa oficina para a prevenção, detecção precoce, diagnóstico, tratamento e cuidados paliativos no câncer de mama e aponta possíveis estratégias que devem ser utilizadas para a sua implementação no Sistema Ú nico de Saúde.

\section{Prevenç̃o}

Embora tenham sido identificados alguns fatores ambientais ou comportamentais associados a um risco aumentado de desenvolver o câncer de mama, estudos epidemiológicos não fornecem evidências conclusivas que justifiquem a recomendação de estratégias específicas de prevenção.

É recomendação que alguns fatores de risco, especialmente a obesidade e o tabagismo, sejam alvo de ações visando à promoção à saúde e a prevenção das doenças crônicas não-transmissíveis, em geral.

$\mathrm{N}$ ão há consenso de que a quimioprofilaxia deva ser recomendada às mulheres assintomáticas, independente de pertencerem a grupos com risco elevado para 0 desenvolvimento do câncer de mama.

\section{DeteCção PRECOCE}

Para a detecção precoce do câncer de mama recomenda-se:

- Rastreamento anual, por meio do exame clínico da mama, em todas as mulheres a partir de 40 anos de idade. Este procedimento é ainda compreendido como parte do atendimento integral à saúde da mulher, e deve ser realizado em todas as consultas clínicas, independente da faixa etária;

- Rastreamento por mamografia em mulheres com idade 
entre 50 a 69 anos, com o intervalo máximo de dois anos entre os exames;

- Exame clínico da mama e mamografia anual, a partir dos 35 anos, nas mulheres pertencentes a grupos populacionais com risco elevado de desenvolver câncer de mama;

- Garantia de acesso ao diagnóstico, tratamento e seguimento para todas as mulheres com alterações nos exames realizados.

São definidos como grupos populacionais com risco elevado para o desenvolvimento do câncer de mama:

- Mulheres com história familiar de pelo menos um parente de primeiro grau (mãe, irmã ou filha) com diagnóstico de câncer de mama, abaixo dos 50 anos de idade;

- Mulheres com história familiar de pelo menos um parente de primeiro grau (mãe, irmã ou filha) com diagnóstico de câncer de mama bilateral ou câncer de ovário, em qualquer faixa etária;

- Mulheres com história familiar de câncer de mama masculino;

- Mulheres com diagnóstico histopatológico de lesão mamária proliferativa com atipia ou neoplasia lobular in situ.

\section{Diagnóstico}

\section{Exame clínico}

0 exame clínico da mama (ECM) é parte fundamental da propedêutica para o diagnóstico de câncer. D eve ser realizado como parte do exame físico e ginecológico, e constitui a base para a solicitação dos exames complementares. Como tal, deve contemplar os seguintes passos para sua adequada realização: inspeção estática e dinâmica, palpação das axilas e palpação da mama com a paciente em decúbito dorsal.

\section{Diagnóstico das lesôes palpáveis}

A ultra-sonografia (USG ) é o método de escolha para avaliação por imagem das lesões palpáveis em mulheres com menos de 35 anos. $N$ aquelas com idade igual ou superior a 35 anos, a mamografia é o método de eleição. Ela pode ser complementada pela ultra-sonografia nas seguintes situações:

- N ódulo sem expressão, porque a mama é densa ou porque está em zona cega na mamografia;

- N ódulo regular ou levemente lobulado, que possa ser um cisto;

- D ensidade assimétrica difusa, que possa ser lesão sólida, cisto ou parênquima mamário.
A ultra-sonografia complementar não deve ser solicitada nas lesões Categoria 2 e 5 (BI-RAD $®$ ), microcalcificações, distorção da arquitetura e densidade assimétrica focal.

Se houver lesões suspeitas, deve-se buscar a confirmação do diagnóstico, que pode ser citológico, por meio de punção aspirativa por agulha fina (PAAF), ou histológico, quando o material for obtido por punção, utilizando-se agulha grossa (PAG) ou biópsia cirúrgica convencional.

A PAAF é um procedimento ambulatorial, de baixo custo, de fácil execução e raramente apresenta complicações, que permite o diagnóstico citológico das lesões. Esse procedimento dispensa o uso de anestesia.

A PAG a ou core biopsy é também um procedimento ambulatorial, realizado sob anestesia local, que fornece material para diagnóstico histopatológico (por congelação, quando disponível), permitindo inclusive a dosagem de receptores hormonais.

$\mathrm{N}$ as lesões palpáveis com imagem negativa (mamografia eultra-sonografia), prosseguir a investigação com PAAF, PAG ou biópsia cirúrgica. H avendo indisponibilidade da realização de exames de imagem, está indicada a investigação por meio da PAAF ou PAG.

0 diagnóstico prévio reduz o estresse da mulher quanto ao conhecimento do procedimento cirúrgico a que será submetida, otimiza o planejamento das atividades do centro cirúrgico, além de ser de custo inferior quando comparado a uma internação para biópsia cirúrgica convencional.

\section{Diagnóstico das lesões não palpáveis}

A conduta nas lesões não pal páveis segue a proposta do Breast I maging Reporting and D ata System (BIRADS $\left.{ }^{\circ}\right)$, publicado pelo Colégio Americano de Radiologia (ACR) erecomendada pelo Colégio Brasileiro de Radiologia (CBR), em reunião de Consenso em1998. A edição de 2003 do BI-RADS ${ }^{\circledR}$ está disponível na Internet, na página do ACR 〈http:// www.acr.org>

$\mathrm{N}$ os casos Categoria 3 (BI-RADS ${ }^{\circledR}$ ), devem ser realizados dois controles radiológicos com intervalo semestral, seguidos de dois controles com intervalo anual.

$\mathrm{N}$ as lesões Categoria 4 e 5 (BI-RADS $\left.{ }^{\circledR}\right)$, está indicado estudo histopatológico, que pode ser realizado por meio de PAG, mamotomia ou biópsia cirúrgica.

Por tratar-se de lesão não pal pável, a biópsia cirúrgica deve ser precedida de marcação (M PC, marcação pré cirúrgica), que pode ser guiada por raios $X$ (mão livre, biplanar ou estereotaxia) ou por ultra-sonografia.

\footnotetext{
a Este procedimento não estava disponível na tabela do SUS até abril de 2004, data de publicação deste documento.
} 
PAG e mamotomia podem ser guiadas por raios $X$ (estereotaxia) ou por ultra-sonografia.

Se houver disponibilidade, recomenda-se eleger a USG para guia de procedimento invasivo quando a lesão for perceptível pelos dois métodos.

Nos casos de PAG e mamotomia com resultado histopatológico benigno, é necessário saber como foi 0 procedimento para decidir a conduta.

0 procedimento (PAG, mamotomia) é considerado adequado se produzir fragmentos íntegros (mínimo de três) e se a lesão for atingida.

$\mathrm{N}$ as lesões Categoria 4 (BI-RADS $®$ ), nos casos de procedimento adequado deve-se fazer dois controles radiológicos com intervalo semestral, seguidos de dois controles com intervalo anual; nos casos não adequados, indicar biópsia cirúrgica.

$N$ as lesões $C$ ategoria 5 (BI-RADS $®$ ), se o resultado histopatológico for benigno, deve-se proceder à investigação com biopsia cirúrgica, tanto nos casos de procedimento adequado quanto nos casos de procedimento não adequado.

A biópsia cirúrgica também está indicada nos casos de exame histopatológico radial scar, hiperplasia atípica, carcinoma in situ, carcinoma microinvasor e material inadequado, quando a biópsia for realizada em material obtido por meio PAG ou mamotimia.

A correlação entre análise do procedimento e conduta está resumida no Q uadro 1.

Quadro 1: Conduta nos casos discordantes, lesões Categoria 4 e 5 (BI-RADS).

\begin{tabular}{|c|c|c|c|}
\hline Categoria & Resultado da PAG ou MT & Análise do procedimento & Indicação \\
\hline 4 & benigno & PAG ou MT adequada & $\begin{array}{c}\text { controle } \\
\text { mamográfico em } \\
6 \mathrm{~m}, 6 \mathrm{~m} \text { e } 1 \text { ano. }\end{array}$ \\
\hline 4 & benigno & PAG ou MT não adequada & biópsia cirúrgica \\
\hline 4 & $\begin{array}{l}\text { carcinoma in situ, radial scar, } \\
\text { hiperplasia atípica ou } \\
\text { se o patologista } \\
\text { solicitar mais material }\end{array}$ & $\begin{array}{l}\text { PAG ou MT adequada } \\
\text { PAG ou MT não adequada }\end{array}$ & biópsia cirúrgica \\
\hline 4 & carcinoma infiltrante & $\begin{array}{l}\text { PAG ou MT adequada } \\
\text { PAG ou MT não adequada }\end{array}$ & tratamento \\
\hline 5 & benigno & $\begin{array}{l}\text { PAG ou MT adequada } \\
\text { PAG ou MT não adequada }\end{array}$ & biópsia cirúrgica \\
\hline 5 & $\begin{array}{c}\text { carcinoma in situ, radial scar, } \\
\text { hiperplasia atípica ou } \\
\text { se o patologista } \\
\text { solicitar mais material }\end{array}$ & $\begin{array}{l}\text { PAG ou MT adequada } \\
\text { PAG ou MT não adequada }\end{array}$ & biópsia cirúrgica \\
\hline 5 & carcinoma infiltrante & $\begin{array}{l}\text { PAG ou MT adequada } \\
\text { PAG ou MT não adequada }\end{array}$ & Tratamento \\
\hline
\end{tabular}

PAG = punção com agulha grossa (core biópsia); M T = mamotomia

\section{Diagnóstico citopatológico}

O s critérios citológicos para a avaliação das lesões mamárias podem ser categorizados como:

- padrão citológico benigno, negativo para malignidade; - padrão citológico positivo para malignidade - apresenta celularidadealta, com células epiteliais atípicas, geralmente isoladas e com citoplasma intacto, ausência de núcleos nus e redução da coesão celular. Sempre que possível acompanha a especificação diagnóstica do processo;

- padrão citológico de malignidade indeterminada (tumor papilar, tumor filóide);
- padrão citológico suspeito para malignidade (lesão epitelial proliferativa com atipias).

\section{Diagnóstico histopatológico}

0 relatório histopatológico deve conter todos os elementos necessários para o adequado manuseio clínico da paciente sob o ponto de vista prognóstico e terapêutico, apresentando a descrição das características da neoplasia, do estado linfonodal, do comprometimento das margens cirúrgicas de ressecção e o resultado dos marcadores prognósticos avaliados por imunoistoquímica (ANEXO 1). 


\section{Estadiamento}

0 estadiamento do câncer de mama é baseado na classificação dos Tumores M alignos, T N M, proposta pela União Internacional C ontra o Câncer, UICC, conforme as características do tumor primário, dos linfonodos das cadeias de drenagem linfática do órgão em que o tumor se localiza, e a presença ou ausência de metástases à distância (ANEXO 2).

\section{Tratamento}

O câncer demama deveser abordado por uma equipe multidisplinar visando o tratamento integral da paciente. As modalidades terapêuticas disponíveis atualmente são a cirúrgica e a radioterápica para o tratamento locoregional, e a hormonioterapia e a quimioterapia para 0 tratamento sistêmico.

\section{Cirurgia}

A indicação de diferentes tipos de cirurgia depende do estadiamento clínico e do tipo histológico, podendo ser conservadora, fazendo-se a ressecção de um segmento da mama (engloba a setorectomia, a tumorectomia alargada e a quadrantectomia) com retirada dos gânglios axilares ou linfonodo sentinela, ou não-conservadora (mastectomia).

São modalidades de mastectomia:

- M astectomia simples ou total (retirada da mama com pele e complexo aréolo-papilar);

- M astectomia com preservação de um ou dois músculos peitorais acompanhada de linfadenectomia axilar (radical modificada);

-M astectomia com retirada do(s) músculo(s) peitoral(is) acompanhada de linfadenectomia axilar (radical);

- M astectomia com reconstrução imediata;

- M astectomia poupadora de pele.

\section{Carcinoma ductal in situ}

A mastectomia simples é um tratamento curativo em $98 \%$ dos casos, mas certamente representa procedimento excessivamente mutilante para considerável parcela dos casos. Tumores com diâmetro inferior a dois centímetros e margens cirúrgicas livres de comprometimento podem ser tratados com ressecção segmentar seguida de radioterapia complementar.

Cirurgias não conservadoras da mama, seguidas ou não de reconstrução mamária, são indicadas quando é impossível assegurar a obtenção de margens livres, em função da extensão ou multicentricidade do tumor. A linfadenectomia de nível I (base da axila) ou a dissecção do linfonodo sentinela deve ser realizada em casos de comedonecrose ou alto grau histológico (GIII), devido à possibilidade de microinvasão e envolvimento axilar.

$\mathrm{N}$ os carcinomas ductais in situ pode-se ainda considerar a possibilidade do uso de hormonioterapia adjuvante com tamoxifeno (20mg/dia), por cinco anos.

$\mathrm{N}$ a neoplasia lobular in situ, considerada como um fator de risco para o desenvolvimento do câncer de mama, indica-se a biópsia excisional. Esta condição exige vigilância especial por meio de exame clínico semestral e mamografia anual.

Carcinomas invasores com diâmetro tumoral inferior a três centímetros

A cirurgia conservadora preenche os prérequisitos que norteiam o tratamento cirúrgico do câncer de mama, que são: máximo controle loco-regional, estadiamento, prognóstico semelhante com menor morbidade e mutilação.

São pré-requisitos para se indicar uma cirurgia conservadora:

- realização de mamografia prévia;

- diâmetro tumoral menor que $3 \mathrm{~cm}$;

- ausência de comprometimento da pele;

- tumor único;

- avaliação das margens cirúrgicas (no intra ou pósoperatório);

- proporção adequada entre volume da mama e do tumor (distorção menor do que 30\%);

- facilidade de acesso ao sistema de saúde para garantia do seguimento.

A avaliação das margens cirúrgicas modifica a extensão da cirurgia e contribui para reduzir a incidência de recidiva local nas cirurgias conservadoras. Q uando a avaliação das margens for feita posteriormente à cirurgia e for identificado o comprometimento das mesmas, recomenda-se a re intervenção. À cirurgia conservadora, segue-se a radioterapia complementar na mama.

$\mathrm{N}$ as mulheres com tumor de diâmetro igual ou inferior a três centímetros, sem comprometimento nodal clinicamente aparente, pode ser utilizada, quando disponível, a técnica do linfonodo sentinela (LS) que visa identificar aquelas que necessitam ser submetidas à linfadenectomia axilar. É baseada na retirada do LS, que é o primeiro linfonodo a receber células metastáticas do tumor primário. 0 LS pode ser identificado pelo cirurgião por meio de injeção de corantes vitais e/ou radiofármacos, seguido de linfocintigrafia e uso de detector portátil de irradiação (probe). 0 exame citológico ou histológico érealizado pelo patologista para identificar ou descartar a presença de metástases. Esta técnica deve ser realizada por equipe multidisciplinar treinada (mastologista, patologista e médico nuclear).

Constituem contra-indicações para a realização da técnica do LS: realização de biópsia prévia complicada 
por hematoma, presença de cicatrizes extensas, mamoplastia, quimioterapia neoadjuvante e gravidez.

$\mathrm{N}$ ão havendo disponibilidade da técnica do LS ou nos casos em que o LS mostrou-se comprometido pela neoplasia maligna da mama, deve-se proceder à linfadenectomia axilar.

Carcinomas invasores com diâmetro igual ou maior que três centímetros, com ou sem quimioterapia neoadjuvante

A mastectomia deve ser indicada para os tumores iguais ou maiores que três centímetros. As técnicas modificadas que preservam um ou ambos os músculos são as mais empregadas, pois além de assegurarem resultados semelhantes à mastectomia radical, facilitam a reconstrução e reduzem a morbidade. A opção pela técnica depende dos achados intra-operatórios, das circunstâncias clínicas e da idade da paciente. Sempre que se indicar uma mastectomia em pacientes com boas condições clínicas, deve-se considerar a possibilidade de se realizar a reconstrução mamária imediata.

\section{Radioterapia}

É utilizada com o objetivo de destruir as células remanescentes após a cirurgia ou para reduzir o tamanho do tumor antes da cirurgia. Após cirurgias conservadoras, deve ser aplicada em toda a mama da paciente, independente do tipo histológico, idade, uso de quimioterapia ou hormonioterapia ou mesmo com as margens cirúrgicas livres de comprometimento neoplásico.

0 reforço da dose no leito tumoral (boost), está indicado nas pacientes com carcinoma ductal infiltrante, principalmente naquelas com idade inferior a 50 anos. O s casos de carcinoma ductal in situ, quando tratados por meio da cirurgia conservadora, devem ser submetidos à radioterapia adjuvante em toda a mama.

A presença de um dos fatores listados a seguir é suficiente para a indicação de radioterapia após a mastectomia, conforme o consenso alcançado no encontro de St. Gallen, Suíça:

- tumores com diâmetro igual ou maior que cinco centímetros (somar com o tamanho do fragmento de biópsia prévia);

- pele comprometida pelo tumor;

- dissecção inadequada da axila;

- margem comprometida (menor do que $1 \mathrm{~cm}$.);

- quatro ou mais linfonodos comprometidos;

- não houve consenso quanto a indicação derealizar radioterapia quando há de um a três linfonodos comprometidos.

A utilização de quimioterapia com antracíclicos no período após as cirurgias radicais ou conservadoras protela o início da radioterapia para o término da terapêutica sistêmica, não devendo ser este período superior a seis meses desde a realização da cirurgia.

Existindo mais de quatro linfonodos axilares comprometidos e em tumores centrais ou mediais, pode se incluir a cadeia da mamária interna, principalmente nos três primeiros espaços intercostais, uma vez que a possibilidade de comprometimento é de $30 \%$.

\section{Quimioterapia e hormonioterapia}

A terapia adjuvante sistêmica segue-se ao tratamento cirúrgico instituído. Sua recomendação deve basear-se no risco de recorrência. Para auxiliar nessa decisão, pode ser utilizado um dos programas obtidos pela internet, nos seguintes endereços: বhttp://www.adjuvantonline.com>e «tttp://www.mayoclinic.com/calcs/>

As mulheres com indicação de mastectomia como tratamento primário, podem ser submetidas à quimioterapia neoadjuvante, seguida de tratamento cirúrgico conservador, complementado por radioterapia. Para aquelas que apresentarem receptores hormonais positivos, a hormonioterapia, também está recomendada.

\section{Estádios I, II e III (operável)}

As pacientes consideradas com risco mínimo de recorrência devem ser submetidas a seguimento periódico. Para aquelas pacientes com risco baixo devese usar Tamoxifeno (T M X) por cinco anos. Já naquelas com risco elevado, o tratamento será condicionado à avaliação dos seguintes fatores: capacidade de resposta aos hormônios, presença de menopausa e comprometimento nodal, como mostrado nos quadros a seguir (quadros 2 e 3 ).

$\mathrm{N}$ ão há indicação, no momento, para a substituição do Tamoxifeno por inibidor de aromatase. $N$ os casos de contra-indicação para uso de TMX, como na ocorrência de doença tromboembólica, doença cerebrovascular ou carcinoma de endométrio e naqueles tumores iniciais que se desenvolvam durante uso de $T M X$, sugere-se inibidor de aromatase como terapia adjuvante somente em mulheres na pós-menopausa e com tumores positivos para receptores hormonais.

$\mathrm{H}$ á poucos estudos que avaliam o impacto da quimioterapia adjuvante em mulheres com idade superior a 70 anos. A sua utilização nesta faixa etária deve ser feita de forma criteriosa e individualizada.

\section{Estádio III (não-operável)}

No tratamento neoadjuvante está recomendado 0 uso de Antracíclico (até 6 ciclos) ou CM F. Na impossibilidade da administração de quimioterapia, a hormonioterapia deve ser instituída. Recomenda-se 0 uso de TMX por um período de quatro a seis meses. 
Em caso de falha no uso de Antracíclico, deve-se adotar a radioterapia acrescida de hormonioterapia se o tumor for receptor positivo.

$\mathrm{N}$ ão houve consenso sobre o uso neoadjuvante dos Taxanes e seus benefícios para as mulheres com câncer de mama, uma vez que, são necessários maiores estudos a seu respeito.

0 tratamento adjuvante consiste no uso de Tamoxifeno, por 5 anos, em pacientes com tumores positivos para receptores hormonais.

Quadro 2: Tratamento de pacientes nos estádios I, II e III (doença operável) conforme o risco de recorrência

\begin{tabular}{|c|c|c|}
\hline $\begin{array}{c}\text { RISCO DE } \\
\text { RECORRÊNCIA }\end{array}$ & CARACTERÍSTICAS & RECOMENDAÇÕES \\
\hline Mínimo & $\begin{array}{l}\text { Idade superior a } 35 \text { anos e } \\
\text { Tumor menor que } 1 \mathrm{~cm} \text { e } \\
\text { Linfonodo axilar negativo ou } \\
\text { Tumor tubular ou mucinoso ou medular típico ou } \\
\text { papilífero menor do que } 3 \mathrm{~cm} \text { e Linfonodo negativo }\end{array}$ & Seguimento periódico \\
\hline Baixo & $\begin{array}{l}\text { Idade superior a } 35 \text { anos e } \\
\text { Tumor entre } 1 \text { e } 2 \mathrm{~cm} \text { e } \\
\text { Grau I ou grau II e } \\
\text { Receptor hormonal (estrogênio e/ou progesterona) } \\
\text { positivo e } \\
\text { Linfonodo negativo }\end{array}$ & $T M X$, por cinco anos \\
\hline Elevado & $\begin{array}{l}\text { Idade inferior a } 35 \text { anos ou } \\
\text { Tumor maior que } 2 \mathrm{~cm} \text { ou } \\
\text { Grau III ou } \\
\text { Receptor hormonal (estrogênio e progesterona) } \\
\text { negativo ou } \\
\text { Linfonodo positivo }\end{array}$ & Ver quadro 3 \\
\hline
\end{tabular}

Quadro 3: Tratamento de pacientes nos estádios I, II e III (doença operável) com risco elevado de recorrência

\begin{tabular}{|c|c|c|}
\hline Condições & Pré-menopausa & Pós-menopausa \\
\hline \multicolumn{3}{|c|}{ Hormônio responsivo } \\
\hline Com linfonodo axilar negativo & $\begin{array}{l}\text { Antracíclico (até } 6 \text { ciclos) ou CMF } \\
\text { seguidos de Tamoxifeno por } 5 \text { anos }\end{array}$ & \multirow{2}{*}{$\begin{array}{c}\text { TMX por } 5 \text { anos ou } \\
\text { Antracíclico (até } 6 \text { ciclos) } \\
\text { seguido de TMX por } 5 \\
\text { anos }\end{array}$} \\
\hline Com linfonodo axilar positivo & $\begin{array}{c}\text { Antracíclico (até } 6 \text { ciclos) seguido de } \\
\text { TMX por } 5 \text { anos }\end{array}$ & \\
\hline \multicolumn{3}{|c|}{ Hormônio não responsivo } \\
\hline Com linfonodo axilar negativo & \multirow{2}{*}{ Antracíclico (até 6 ciclos) } & \multirow{2}{*}{ Antracíclico (até 6 ciclos) } \\
\hline Com linfonodo axilar positivo & & \\
\hline
\end{tabular}

Estádio IV (terapia paliativa)

A doença metastática deve ser confirmada cito ou histologicamente, sempre que possível.

A indicação hormonioterapia isolada deve ocorrer sempre que possível. D eve ser utilizada somente em tumores com receptor hormonal positivo, conforme descrito no Q uadro 4.
A Q uimioterapia está recomendada nos tumores negativos para receptores hormonais, nos casos de doença visceral sintomática ou extensa e quando há progressão após intervalo curto de manipulação hormonal prévia (4 meses), devendo ser realizada conforme indicado no Q uadro 5. 
Quadro 4: Recomendações para hormonioterapia em pacientes no estádio IV

\begin{tabular}{|c|c|c|}
\hline Hormonioterapia & Pré-menopausa & Pós-menopausa \\
\hline $1^{\underline{a}}$ linha & Ablação ovariana $^{(1)}$ ou TMX & TMX \\
\hline $2^{\underline{a}}$ linha & TMX isolado $^{(2)}$ ou ablação ovariana isolada & Inibidor de aromatase $^{(4)}$ \\
\hline $3^{\underline{a}}$ linha $^{a}$ & Inibidor de aromatase $^{(3)}$ & Acetato de megestrol \\
\hline $4^{\underline{a}}$ linha $^{b}$ & Acetato de megestrol & - \\
\hline
\end{tabular}

N otas:

(1) A ablação ovariana pode ser realizada por cirurgia ou radioterapia.

(2) A terapêutica hormonal de segunda linha, na pré-menopausa, fica na dependência da opção adotada como 1ạ linha.

(3) A utilização de inibidores de aromatase deve ser feita somente em mulheres na pós-menopausa ou, se na pré-menopausa, apenas naquelas em que foi realizada ablação ovariana.

(4) Não há evidências de aumento de sobrevida com a utilização de inibidores de aromatase na primeira linha do tratamento paliativo de mulheres na pós-menopausa, com câncer de mama receptor hormonal positivo.

Quadro 5: Recomendações para quimioterapia em pacientes no estádio IV

\begin{tabular}{|c|c|}
\hline Quimioterapia & Drogas recomendadas \\
\hline 19 linha & CMF ou FAC \\
\hline $2^{\mathrm{a}}$ linha & $\begin{array}{c}\text { Taxanes ou Capecitabina ou } \\
\text { Vinorelbine }\end{array}$ \\
\hline $3^{\mathrm{a}}$ linha & $\begin{array}{l}\text { Taxanes ou Capecitabina ou } \\
\text { Vinorelbine, desde que não tenha } \\
\text { sido usada como } 2^{\text {a }} \text { linha }\end{array}$ \\
\hline
\end{tabular}

N otas:

1. A escolha de quimioterapia paliativa de primeira linha está na dependência de tratamento adjuvante prévio e do intervalo entre a sua administração e a recorrência da doença. Em caso de intervalo superior a um ano, pode-se considerar reinstituição do mesmo esquema quimioterápico. Entretanto, a reutilização de esquema terapêutico com Adriamicina estará vinculada à dose prévia utilizada, respeitando-se 0 limite total de $450 \mathrm{mg} / \mathrm{m} 2$.

2. Considerar a quimioterapia de terceira linha, desde que anteriormente tenham sido empregados CM F e Adriamicina.

3. 0 uso da quimioterapia paliativa deve ser adotada por no máximo 6 meses em cada linha.

Há evidências recentes de aumento de sobrevida com utilização precoce de Trastuzumab associado à quimioterapia, em pacientes com tumores que superexpressam HER-2. No entanto, a sua utilização está condicionada a estudos de avaliação econômica a serem realizados pelo M inistério da Saúde.

\section{Seguimento}

No seguimento das pacientes com câncer de mama após tratamento adjuvante devese proceder à história, exame físico, mamografia e exame ginecológico conforme descrito no quadro a seguir (quadro 6). N ão há indicação de realização de hemograma, bioquímica no sangue, radiografia de tórax, ultra-sonografia abdominal ou trans-vaginal, cintilografia óssea e marcadores tumorais na ausência de sintomas ou de indicações clínicas que justifiquem sua solicitação.

Quadro 6: Recomendações para o seguimento de mulheres com câncer de mama

\begin{tabular}{|l|c|c|}
\hline \multirow{2}{*}{ Recomendação } & \multicolumn{2}{c|}{ Tempo decorrido } \\
\cline { 2 - 3 } & $1-5$ anos & Após o 5ㅇaㅇ ano \\
\hline História e Exame físico & semestral & anual \\
\hline Mamografia & anual & anual \\
\hline $\begin{array}{l}\text { Exame ginecológico } \\
\text { (quando em uso de } \\
\text { Tamoxifeno) }\end{array}$ & anual & anual \\
\hline
\end{tabular}

\section{INTERVENÇÕES INTERDISCIPLINARES}

As ações interdisciplinares na atenção ao câncer de mama devem ser iniciadas a partir do diagnóstico, e devem fazer parte da atuação conjunta entre todos os profissionais de saúde, junto aos pacientes e familiares.

As intervenções interdisciplinares têm como objetivo fazer uma junção entre conhecimentos e disciplinas, que intercedam efetivamente na qualidade de vida desta população após o tratamento, favorecendo de forma prioritária o seu retorno às atividades físicas, sociais e profissionais.

A equipe interdisciplinar deverá ser composta por: médico, enfermeiro, psicólogo, fisioterapeuta, terapeuta ocupacional, assistente social e nutricionista.

\footnotetext{
${ }^{a}$ A hormonioterapia neoadjuvante em câncer de mama estádio III (não operável), não estava disponível na Tabela do SU S até abril de 2004, data da publicação deste documento

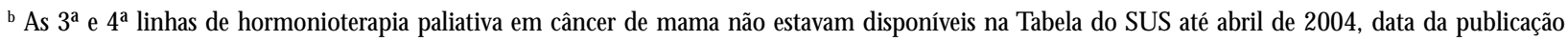
deste documento.
} 
A atuação interdisciplinar para a prevenção de complicações decorrentes do tratamento deve ser realizada em todas as fases: diagnóstico; durante e após o tratamento; na recorrência da doença e nos cuidados paliativos. Em cada uma dessas fases, é necessário conhecer e identificar as necessidades da mulher, os sintomas e suas causas, e o impacto destes no seu cotidiano.

A intervenção consiste em orientações domiciliares, tratamento ambulatorial, tratamento hospitalar específicos e grupos educativos. D eve ser garantido 0 acesso às informações relacionadas aos direitos previstos em lei e adequação dos recursos que garantam uma atenção integral.

\section{Psicologia}

0 atendimento psicológico deve ser iniciado imediatamente após o diagnóstico e definição da conduta terapêutica oncológica, estabelecendo-se uma avaliação psicológica individual para o planejamento dos atendimentos subseqüentes, que poderão ser em grupo.

Q uando não for possível a reconstrução mamária, próteses mamárias de uso externo devem ser adaptadas após a alta do curativo, visando diminuir a alteração na imagem corporal.

\section{Enfermagem}

A atuação do enfermeiro deve ser iniciada logo após o diagnóstico, por meio da consulta de enfermagem, a ser realizada por ocasião da internação e antes de cada modalidade terapêutica. No pós-operatório deve-se avaliar a ferida operatória e orientar para a alta, direcionando a mulher para o autocuidado (cuidados com o sítio cirúrgico, dreno e membro homolateral).

No momento da alta hospitalar deve-se encaminhar a mulher para grupos de apoio interdisciplinar, que discutem aspectos educativos, sociais e emocionais, visando à reintegração à vida cotidiana. Por fim, no seguimento ambulatorial da ferida operatória deve-se avaliar e realizar os curativos, retirar dreno, realizar punção de seroma e acompanhar a mulher durante todo o período de cicatrização.

\section{Fisioterapia}

A atuação do fisioterapeuta deve ser iniciada no pré operatório, objetivando conhecer as alterações préexistentes e identificar os possíveis fatores de risco para as complicações pós-operatórias. Q uando necessário, deve ser instituído tratamento fisioterapêutico já nesta etapa, visando minimizar e prevenir as possíveis seqüelas. No pós-operatório imediato, objetiva-se identificar alterações neurológicas ocorridas durante 0 ato operatório, presença de sintomas ál gicos, edema linfático precoce e alterações na dinâmica respiratória.

D urante a terapia adjuvante e no seguimento, devese priorizar a prevenção e minimização das complicações, sejam elas linfáticas, posturais, funcionais e/ou respiratórias. As recomendações para a prevenção, diagnóstico e tratamento do linfedema são apresentadas no ANEXO 3.

Para o controle dos sintomas álgicos, as pacientes devem realizar exercícios domiciliares, manobras ativas de relaxamento muscular e automassagem no local cirúrgico. A atividade física deve ser recomendada, sendo contra-indicado o uso do braço em movimentos rápidos e de repetição, assim como atividades com carga.

\section{CUIDADOS PALIATIVOS}

Segundo a definição da O rganização M undial de Saúde, "Cuidados Paliativos consistem na assistência promovida por uma equipe multidisciplinar, ativa e integral a pacientes cuja doença não responde mais ao tratamento curativo, sendo o principal objetivo a garantia da melhor qualidade de vida, tanto para o paciente quanto para seus familiares, através do controle da dor e demais sintomas, em suas dimensões psicossociais e espirituais".

Sua instituição deve ser precoce, à época do diagnóstico de doença avançada, sem possibilidade de cura. A transição do cuidado ativo para o cuidado com intenção paliativa é um processo dinâmico e diferente em cada paciente.

Os Cuidados Paliativos visam garantir melhor qualidade de vida, controle da dor e demais sintomas, além de facilitar a desospitalização. D evem ainda contribuir para reduzir a realização de exames complementares quando seus resultados não mudam a terapia, evitar 0 uso de terapias ineficazes e potencialmente danosas aos pacientes, enfatizar 0 tratamento domiciliar em detrimento do tratamento hospitalar, preparar os cuidadores para a realização, em ambiente domiciliar, de cuidados antes restritos às instituições e estruturar 0 acesso à distribuição e à dispensação de insumos e medicamentos necessários à manutenção do paciente no seu domicílio.

A prática clínica de cuidados paliativos segue princípios éticos baseados no respeito à autonomia do paciente, requer habilidade de comunicação e uma abordagem interdisciplinar.

A equipe interdisciplinar deve ser composta por médico, enfermeiro, fisioterapeuta, assistente social, psicólogo, nutricionista e terapeuta ocupacional, sendo cada profissional responsável pelas condutas dentro de 
sua área de competência. Tais condutas, no entanto, devem sempre ser discutidas com toda a equipe. É ainda importante a atuação do voluntário junto à equipe.

A habilidade de comunicação tem um importante papel, uma vez que a informação e explicação adequadas usualmente diminuem a ansiedade dos pacientes e familiares, estabelecendo-se um sentimento de confiança e segurança na equipe.

No ANEXO 4 são apresentados os principais aspectos relacionados ao controle dos sintomas, com destaque para astenia, síndrome anorexia / caquexia, lesões tumorais da pele, dor, dispnéia, alterações neurológicas / psiquiátricas, depressão maior e delírio.

\section{SOCIEDADE CIVIL ORGANIZADA}

Sua participação nas ações de controle do câncer de mama tem papel fundamental no fortalecimento do controle social e na capacidade de mobilização em torno de projetos de responsabilidade social, que começam a fazer parte da agenda empresarial. D essa forma cumpre o seu papel de núcleo mobilizador de parcerias em prol da humanização da saúde.

Foram recomendadas as seguintes estratégias: - Criação de um Grupo Permanente de Trabalho, composto por diversas organizações que reconhecidamente vêm desenvolvendo ações na área de câncer de mama, com a presença das sociedades científicas afins, com o objetivo de colaborar e monitorar a implantação das ações de controle do câncer de mama no País. Esse grupo participará diretamente na proposição e execução de ações de educação comunitária e continuada para os profissionais, visando promover a humanização da assistência, na perspectiva dos direitos humanos.

- Realização de seminários regionais, de capacitação de lideranças comunitárias e de conselheiros municipais (saúde, educação e assistência social), enfatizando o controle social, culminando em um Encontro $\mathrm{N}$ acional de Luta no Combate ao C âncer de $M$ ama.

- Articulação com os Institutos de Direitos do Consumidor, Ministério Público, Ordem dos Advogados do Brasil e a Comissão Intersetorial da Saúde da Mulher, com o objetivo de garantir os direitos da mulher no que diz respeito ao diagnóstico precoce e à reconstrução mamária. As mulheres deverão ter acesso à informação por meio de cartilha informativa sobre seus direitos.

- Estimular a criação de grupos de ajuda nos hospitais de referência para as mulheres com câncer de mama.

\section{RECOMENDAÇÕES PARA O SUS}

- Estimular, mediante ações educativas nos meios de comunicação, o exame físico da mama realizado pelo profissional de saúde.

- D esenvolver ações de educação para o ensinamento da palpação das mamas pela própria mulher como estratégia dos cuidados com o próprio corpo. 0 exame realizado pela própria mulher não substitui o examefísico por profissional de saúde capacitado para esta atividade. - Implantar o rastreamento mamográfico no país, com garantia do diagnóstico, tratamento em tempo hábil e seguimento das mulheres com alterações mamárias.

- Elaborar e implantar normas técnico-operacionais para a estruturação da rede de saúde e a definição dos fluxos assistenciais visando subsidiar os gestores municipais e estaduais.

- Implantar sistema de informação nos serviços de mamografia credenciados pelo SUS.

- Criar os indicadores e parâmetros assistenciais para cálculo da necessidade de oferta de serviços em todos os níveis de atenção ao câncer de mama.

- Revisar normas técnicas para o credenciamento, o controle e a avaliação dos serviços de mamografia no SU S.

- C riar mecanismos de garantia de qualidade, como parte dos critérios para o credenciamento e monitoramento de serviços de mamografia no SU S.

- Exigir habilitação em mamografia para os médicos responsáveis pela emissão de laudos mamográficos.

- Rever as Portarias M inisteriais (Portaria G M /M S no 2413 de 23 de março de 1998; Portaria GM /M S no 2416 de 23 de março de 1998; Portaria GM/M S no 3535 de 02 de setembro de 1998; Portaria GM /M S no 1319 de 23 de julho de 2002; Portaria SAS/M S no 472 de 24 de julho de 2002; Portaria SAS/M S no 859 de 12 de novembro de 2002) que regulamentam atividades relacionadas aos cuidados paliativos, visando estruturar esta modalidade assistencial na rede SU S.

- Desenvolver uma política de capacitação dos profissionais em todos os níveis de complexidade da atenção ao câncer de mama.

- Inserir conteúdos sobre câncer de mama nos diversos níveis de formação profissional (médio e superior).

- Realizar estudos de avaliação econômica visando a incorporação racional, pelo SU S, de novas tecnologias na área de tratamento de câncer.

- Incorporar a classificação T N M atualizada (UICC, 2002) aos Registros H ospitalares de Câncer e Registros de Câncer de Base Populacional.

- Proceder a revisão da Tabela SU S, a partir de estudos de avaliação econômica, visando analisar a viabilidade de incorporação dos seguintes procedimentos:

- hormonioterapia neoadjuvante em câncer de mama 
estádio III (não operável);

- 3a linha de quimioterapia paliativa;

- 3a e 4a linhas de hormonioterapia paliativa;

-associação de trastuzumab à quimioterapia paliativa em pacientes que superexpressam H ER-2;

\section{BIBLIOGRAFIA}

Baines C J, M iller BA. M ammography versus clinical examination of the breast. M onogr $N$ atl $C$ ancer Inst 1997;22:25-9.

Barton M B, H arris R, Fletcher SW. D oes this patient have breast cancer?T hescreening clinical breast examination: shoud it be done? H ow? JAM A 1999;282:1270-80.

Cuzick J, PowlesT, Veronesi U , Forbes J, EdwardsR, Ashley S, et al. 0 verview of themain outcomes in breast-cancer prevention trials. Lancet 2003;361(9354):296-300.

Fletcher SW, Elmore J G. M amographic screening for breast cancer. N Engl J M ed 2003;348:1672-80.

H unter CP, Redmond CK, Chen VW. B reast cancer: factors associated with stageat diagnosisin black and white women. J $N$ atl C ancer Inst 1993;85:1129-37.

Instituto $\mathrm{N}$ acional de $\mathrm{C}$ âncer (Brasil). Atlas demortalidadepor câncer no Brasil 1979-1999. Rio de Janeiro: IN CA; 2002.

Instituto $\mathrm{N}$ acional deCâncer (Brasil). Câncer no Brasil: dados dos registros de câncer de base populacional, Rio de Janeiro: IN CA; 2003. vol. 3.

Insituto $\mathrm{N}$ acional de Câncer (Brasil). Estimativa da incidência e mortalidadepor câncer no Brasil, 2003. Rio dejaneiro: IN CA;2003.

International Union Against C ancer (U nited States). T N M Classification of malignant tumors. 6th ed. N ew York: John Wiley \& Sons; 2002.

Jackman RJ, N owels K W, Rodriguez-Soto J, M arzoni FA Jr, Finkelstein SI, Shepard M J. Stereotactic, automated large core needle biopsy of nonpal pable breast lesions: false negativeand histologic underestimation rates after long-term follow-up. Radiology 1999;210:799-805.

Koch H A, Azevedo CM , Boechat AL. Radiologia da mama: qualidadeem mamografia. Radiol Bras 1996;29:257-69.

Koch H A, Peixoto JE. Bases para um programa de detecção precoce do câncer demama por meio da mamografia. Radiol Bras 1998;31:329-37.

Lee CH, Philpotts LE, H orvath LJ, Tocino I. Follow-up of breast lesions diagnosed as benign with stereotactic coreneedle biopsy: frequency of mammographic changeand false-negative rate. Radiology 1999;212:189-94.

Liberman L, Feng T L, D ershaw D D , M orris EA, Abramson AF. U S-guided core breast biopsy: use and cost-effectiveness. Radiology 1998;208:717-23.

Miller AB, To T, Baines CJ, Wall C. C anadian national breast screening study-2: 13 year- results of arandomized trial in women aged 50-59 years. J N atl C ancer Inst 2000;92:1490-9.

Miller BA, Feuer EJ, H ankey BF. Recent incidencetrendsfor breast cancer in women and the relevance of early detection: an update. CA Cancer J Clin 1993;43:27-41.

0 Isen $0, G$ otzschePC. Screening for breast cancer with mammography. CochraneD atabaseSyst Rev 2001;(4):CD 001877.

Parker SH , Burbank F, Jackman RJ, Aucreman C J, Cardenosa G, Cink TM , et al. Percutaneous large-core breast biopsy: a multi-institutional study. Radiology 1994;193:359-64.

Parkin D M , Bray FI, D evesa SS. Cancer burden in the year 2000. The global picture. Eur J Cancer 2001;37:S4-66.

Peixoto JE, Koch H A, AlmeidaCD. M anual deinstruções para medidas decontraste de qualidadedos parâmetrostécnicos da mamografia. Rio deJaneiro: Centro deD iagnóstico $\mathrm{M}$ amário da Santa C asa da M isericórdia; 1998.

Tavassoli FA, D evilleP. World H ealth O rganization classification of tumors. Pathology and genetics of tumors of the breast and femalegenital organs. Lyon: IARC; 2003.

Thomas D B, Gao D L, Ray RM , Wang W W, Allison CJ, Chen $\mathrm{FL}$, et al. Randomized trial of breast self-examination in Shangai: final results. J N atl C ancer Inst 2002;94:1445-57.

Weiss N S. B reast cancer mortal ity in relation to clinical breast examination and breast self- examination. Breast J 2003;9 (Suppl 2):S86-9.

W ünsh Filho V, M oncau JE. M ortalidadepor câncer no Brasil 1980-1995: padrõesregionaisetendênciastemporais. Rev Assoc M ed Bras 2002;48:250-7.

\section{ANEXO 1}

\section{DiagnósticO histOPATOLÓGICO}

a) Características da neoplasia

D evem ser consideradas as seguintes características do tumor:

- Tamanho do tumor: Para fins de estadiamento dos tumores por meio da classificação TN M (Tumor, $\mathrm{N}$ ódulo e M etástase), considerar o maior diâmetro do componente invasivo do tumor. A medida macroscópica deve ser confirmada pela medida microscópica. No caso de discrepância, predomina a medida avaliada na microscopia. Em casos de tumores multifocais ou multicêntricos, a medida considerada é a do maior tumor. Deve-se relatar a porcentagem de ductos que contêm o componente in situ.

- Tipo histológico: A classificação do tipo histológico deve seguir a terminologia da O rganização M undial de Saúde, atualizada em 2003.

- Grau histológico: Recomenda-se a utilização do grau histológico combinado de N ottinghan (Scarff, Bloon, Richardson modificado por Elston-Ellis, 1998), que inclui percentual de diferenciação tubular, avaliação do pleomorfismo nuclear e índice mitótico.

- Invasão vascular peritumoral nos vasos sangüíneos ou 
linfáticos: Vasos sanguíneos e linfáticos intra e peritumorais podem ser invadidos por células tumorais. A presença de êmbolos tumorais em vasos linfáticos peritumorais está associada ao aumento de recorrência tumoral e a invasão de vasos sanguíneos correlaciona-se ao desenvolvimento de metástases à distância e pior prognóstico.

b) Estado linfonodal

0 relatório deve contemplar os seguintes itens:

- número de linfonodos dissecados (mínimo de 10);

- número de linfonodos comprometidos;

- tamanho do maior foco metastático;

- invasão capsular e extensão a tecidos extranodais;

- coalescência.

c) Avaliação das margens cirúrgicas de ressecção

As margens cirúrgicas da ressecção devem estar descritas, considerando-se "margem comprometida" a presença de neoplasia na área pintada com tinta nanquim. No caso de "margens livres", designa-se em milímetros, à distância da neoplasia à menor margem.

d) $M$ arcadores prognósticos avaliados por imunoistoquímica

O s receptores hormonais são os principais fatores preditivos do prognóstico de mulheres com câncer de mama. A sua presença se correlaciona com o benefício da terapia hormonal. Recomenda-se a dosagem de receptores de estrógenos e de progesterona em todos os casos, citando-se o percentual de positividade. Considera-se positivo o tumor que apresente receptores em pelo menos $10 \%$ das células.

\section{ANEXO 2}

\section{CLASSIFICAÇÃO TNM}

Esta classificação aplica-se apenas aos carcinomas, sendo indispensável a confirmação histológica. Recomenda-se que, quando houver múltiplos tumores, o maior deles seja considerado para definição dos parâmetros e quando houver tumores sincrônicos bilaterais, a classificação de cada um deles seja isolada.

O s quadros a seguir sintetizam as classificações conforme o tamanho do tumor ( $\mathrm{T}$ ), comprometimento nodal $(N)$ e metástases ( $M)$, além de agrupar, por estádios, as diversas combinações possíveis.

\section{Tamanho do Tumor(T)}

Tx - tumor não pode ser avaliado

Tis - carcinoma in situ

T 1 - tumor com até $2 \mathrm{~cm}$ em sua maior dimensão

T1 mic - carcinoma microinvasor (até $1 \mathrm{~mm}$ )

T 1a - tumor com até $0,5 \mathrm{~cm}$ em sua maior dimensão

$\mathrm{T} 1 \mathrm{~b}$ - tumor com mais de 0,5 e até $1 \mathrm{~cm}$ em sua maior

\section{dimensão}

T 1c - tumor com mais de $1 \mathrm{~cm}$ e até $2 \mathrm{~cm}$ em sua maior dimensão

T2 - tumor com mais de 2 e até $5 \mathrm{~cm}$ em sua maior dimensão

T3 - tumor com mais de $5 \mathrm{~cm}$ em sua maior dimensão T 4 - qualquer T com extensão para pele ou parede torácica

T 4a - extensão para a parede torácica

$\mathrm{T} 4 \mathrm{~b}$ - edema (incluindo peau d'orange), ulceração da pele da mama,

nódulos cutâneos satélites na mesma mama

T 4c - associação do T4a e T4b

$\mathrm{T} 4 \mathrm{~d}$ - carcinoma inflamatório

O bservações:

a. 0 comprometimento do músculo grande peitoral não caracteriza T4.

b. Presença de retração da pele ou papila não interfere no estadiamento.

\section{Linfonodos Regionais (N)}

$\mathrm{Nx}$ - O s linfonodos regionais não podem ser avaliados No - Ausência de metástase

N 1 - Linfonodo(s) homolateral(is) móvel(is) comprometido(s)

N2 - M etástase para linfonodo(s) axilar(es) homolateral (is), fixos uns aos outros ou fixos a estruturas vizinhas ou metástase clinicamente aparente somente para linfonodo(s) da cadeia mamária interna homolateral

N 2a - M etástase para linfonodo(s) axilar(es) homolateral (is) fixo(s) unsaos outros ou fixosa estruturas vizinhas

$\mathrm{N} 2 \mathrm{~b}$ - M etástase clinicamente aparente somente para linfonodo(s) da cadeia mamária interna homolateral, sem evidência clínica de metástase axilar

N 3 - M etástase para linfonodo(s) infraclavicular(es) homolateral(is) com ou sem comprometimento do(s) linfonodo(s) axilar(es), ou para linfonodo(s) da mamária interna homolateral clinicamente aparente na presença de evidência clínica de metástase para linfonodo(s) axilar(es) homolateral (is), ou metástase para linfonodo(s) supraclavicular(es) homolateral(is) com ou sem comprometimento do(s) linfonodo(s) axilar(es) ou da mamária interna

N 3a - M etástase para linfonodo(s) infraclavicular(es) homolateral(is)

N 3b - M etástase para linfonodo(s) da mamária interna homolateral e para linfonodo(s) axilar(es)

N 3c - M etástase para linfonodo(s) supraclavicular(es) homolateral(is)

O bservação: Clinicamente aparente é definido como 
detectado por estudos de imagem (exceto linfocintigrafia), pelo exame clínico ou pelo diagnóstico patológico macroscópico.

\section{Metástases (M)}

Mx - metástase à distância não pode ser avaliada

M 0 - ausência de metástase à distância

M 1 - presença de metástase à distância (incluindo LFN supraclaviculares)

Estadiamento TNM do câncer de mama por agrupamentos

\begin{tabular}{|c|c|}
\hline Estádio 0 & Tis NO MO \\
\hline Estádio I & TI NO MO \\
\hline Estádio II A & $\begin{array}{l}\text { TO N1 MO } \\
\text { T1 N1 MO } \\
\text { T2 NO MO }\end{array}$ \\
\hline Estádio II B & $\begin{array}{l}\text { T2 N1 MO } \\
\text { T3 NO MO }\end{array}$ \\
\hline Estádio III A & $\begin{array}{l}\text { T0 N2 MO } \\
\text { T1 N2 MO } \\
\text { T2 N2 MO } \\
\text { T3 N1 MO } \\
\text { T3 N2 MO }\end{array}$ \\
\hline Estádio III B & $\begin{array}{l}\text { T4 N0 MO } \\
\text { T4 N1 MO } \\
\text { T4 N2 MO }\end{array}$ \\
\hline Estádio III C & Tqq N3 MO* \\
\hline Estádio IV & Tqq Nqq $\mathrm{Ml}^{*}$ \\
\hline
\end{tabular}

\section{ANEXO 3}

\section{Prevenção, diagnóstico e tratamento do linfedema}

A mobilização do braço deve ser limitada a 90 으 de flexão e a abdução de ombro e a rotação externa até 0 limite de tolerância da paciente, para prevenir complicações relacionadas à restrição articular e linfedema, sem aumentar o risco da formação do seroma. Após a retirada dos pontos e do dreno, não havendo intercorrências proibitivas, a mobilização do braço deve ser realizada com amplitude completa. As pacientes devem ser orientadas a adotar uma postura confortável, com o membro superior levemente elevado quando estiverem restritas ao leito.

Para as pacientes submetidas à reconstrução com retalho miocutâneo do músculo reto abdominal, 0 posicionamento adequado é com a cabeceira elevada e semiflexão de joelhos, nas duas primeiras semanas de pós-operatório.

A prevenção do linfedema requer uma série de cuidados, que se iniciam a partir do diagnóstico decâncer de mama. As pacientes devem ser orientadas quanto aos cuidados com o membro superior homolateral à cirurgia, visando prevenir quadros infecciosos e linfedema. Evitar micoses nas unhas e no braço; traumatismos cutâneos (cortes, arranhões, picadas de inseto, queimaduras, retirar cutícula e depilação da axila); banheiras e compressas quentes; saunas; exposição solar; apertar o braço do lado operado (blusas com elástico; relógios, anéis e pulseiras apertadas; aferir a pressão arterial); receber medicações por via subcutânea, intramuscular e endovenosa e coleta de sangue; movimentos bruscos, repetidos e de longa duração; carregar objetos pesados no lado da cirurgia e deitar sobre o lado operado.

Preconiza-se: pele hidratada e limpa; uso de luvas de proteção ao fazer as atividades do lar (cozinhar, jardinagem, lavar louça e contato com produtos químicos); intervalos para descanso durante a execução de atividades de vida diária; utilização de removedor de cutículas ao fazer a unha do lado operado; usar cremes depilatórios, tesoura ou máquina de cortar cabelo na retirada de pelo da axila do lado operado; atenção aos sinais de infecção no braço (vermelhidão, inchaço, calor local); e uso de malhas compressivas durante viagens aéreas.

D eve-se tomar o cuidado para não provocar sensação de incapacidade e impotência funcional. As pacientes devem ser encorajadas a retornarem as atividades de vida diária e devem ser informadas sobre as opções para os cuidados pessoais.

0 diagnóstico do linfedema é obtido através da anamnese e exame físico. O s exames complementares são utilizados quando se objetiva verificar a eficácia de tratamentos ou para analisar patologias associadas.

Considerase linfedema a diferença de pelo menos $2 \mathrm{~cm}$ entre os membros, em um ou mais pontos, obtidos através da perimetria ou volume residual de $200 \mathrm{ml}$ obtido de forma direta (volume de água deslocada) ou indireta (perimetria).

\section{ANEXO 4}

\section{CONTROLE DOS SINTOMAS}

Em relação aos pacientes com câncer de mama avançado, os sintomas de maior freqüência, ressaltando que os demais não devem ser esquecidos, são:

- Astenia. Sintoma mais comum em pacientes com câncer avançado. Seu manejo se dá através do tratamento das causas reversíveis (anemia, infecção, distúrbio hidroeletrolítico, entre outros) e auxílio no estabelecimento de prioridades. 
- Síndrome anorexia / caquexia. É o segundo sintoma mais comum em cuidados paliativos, ocorrendo em 65$85 \%$ dos casos. Seu manejo tem como objetivo a manutenção da integridade física e não a melhora do estado nutricional do paciente.

- Lesões tumorais de pele. Infiltração da pele pelo tumor primário ou metastático com conseqüente desenvolvimento de ulcerações ou lesões "fungóides", causando muitas vezes isolamento social e prejuízo emocional. A terapêutica compreende o tratamento oncológico paliativo (radioterapia e quimioterapia) e tratamento sintomático, possibilitando a regressão da lesão, controle de sangramento, da dor e de infecções secundárias.

- Dor. Sintoma dos mais estudados em cuidados paliativos. Constitui o quinto sinal vital, ocorrendo em $60 \%$ a $90 \%$ dos pacientes com câncer avançado. Sendo assim, torna-se mandatório o uso da "Escala Visual Analógica - EVA" durante a avaliação da dor em paciente com câncer de mama avançado.

Entre as principais causas da dor encontram-se: complicações do próprio tratamento curativo (cirurgia, radioterapia e quimioterapia), metástases ósseas, linfedema e compressão radicular pela doença.

Atualmente, dispõe de grande arsenal terapêutico, incluindo tratamento medicamentoso, quando se segue a Escada Analgésica da O M S, guiando o uso seqüencial de drogas (incluindo os opióides), radioterapia, procedimentos invasivos (bloqueios, cateteres, alcoolização de plexo, D rez [destruição do corno posterior da medula]), acupuntura, psicoterapia, fisioterapia. - D ispnéia. 0 corre em $70 \%$ dos pacientes em cuidados paliativos, sendo que em $24 \%$ dos casos não há causas identificáveis.

Causas relacionadas ao câncer: restrição por invasão da parede torácica, metástases pulmonares, derrame pleural neoplásico, linfangite carcinomatosa.

C ausas relacionadas ao tratamento: fibrose pulmonar por drogas ou radioterapia, comorbidades como doença pulmonar obstrutiva crônica, insuficiência cardíaca congestiva, infecções, tromboembolia pulmonar.

Como medidas de tratamento temos a fisioterapia e 0 tratamento medicamentoso específico.

- Alterações neurológicas/ psiquiátricas. A avaliação inicial das pacientes com câncer de mama avançado e alteração cognitiva inclui exame físico completo e exames laboratoriais. $\mathrm{N}$ ão esquecer que desidratação é a causa mais comum de confusão mental e alteração de comportamento. A realização de exames de imagem é importante quando existe a suspeita de progressão de doença para sistema nervoso central ou a necessidade de exclusão de outras possíveis etiologias dos sintomas.
A radioterapia deve ser realizada na possibilidade de benefício.

- Depressão maior. O s quadros de depressão maior, presente em $20 \%$ dos pacientes em Cuidados Paliativos, e de ansiedade generalizada devem ser tratados com terapia medicamentosa, psicoterapia, estímulo à atividade física e terapia comportamental.

- D elírio. M anifestação neuropsiquiátrica encontrada com freqüência em pacientes com câncer avançado, sendo inclusive descrito na literatura médica mundial como uma das principais indicações de sedação. Entre as possíveis causas destacamos a progressão da doença, incluindo metástases, associação medicamentosa, alterações metabólicas, comorbidades descompensadas. A abordagem deve ser feita visando também o cuidador, que na maioria dos casos encontra-se angustiado e impotente diante da situação.

\section{Instituto $\mathrm{N}$ acional de $\mathrm{C}$ âncer/MS \\ Coordenação de Prevenção e Vigilância}

Gulnar Azevedo e Silva

D ivisão de Atenção 0 ncológica

Roseli M onterro da Silva

D epto de Ações Programáticas e Estratégicas/M S - Área Técnica da Saúde da Mulher

M aria José de O liveira Araújo

Apoio

\section{Sociedade Brasileira de Mastologia}

Ézio Dias N ovaes

Elaboração

Instituto $\mathrm{N}$ acional de $\mathrm{C}$ âncer

Célia Regina Andrade Costa

Fátima M eirelles P. Gomes

Roseli M onteiro da Silva (organizadora)

Revisão

Luiz Claudio Thuler - Área de Avaliação em Saúde

M aria Lucia Brandão - Coordenação de Ensino e

D ivulgação Científica

Produção

Claudia Gomes e Viviane Queiroga - D ivisão de

Comunicação Social

M aria do Carmo Esteves da Costa - D ivisão de Atenção

Oncológica

Colaboradores

Secretaria Estadual de Saúde de Alagoas

M aria Betânia Silva

Secretaria Estadual de Saúde do Amazonas

Luciano Afonso Brandão

Secretaria Estadual de Saúde do D istrito Federal

Edna N akamai

Secretaria Estadual de Saúde do Espírito Santo 
Albertina Salomão Rocha

M orilda T. D umuner de Souza

Secretaria Estadual de Saúde de M inas G erais

Sergio Bicalho

Secretaria Estadual de Saúde da Paraíba

Alana Soares Brandão Barreto

Secretaria Estadual de Saúde do Rio de Janeiro

Risoleide $M$ arques Figueiredo

Secretaria Estadual de Saúde de Santa Catarina

D eise Carvalho Dias

Secretaria Estadual de Saúde de São Paulo

Wilza Vieira Villela

Secretaria Estadual de Saúde de Sergipe

Joana M aria M. Olivera

M aria Augusta Garcez Almeida

Secretaria M unicipal de Saúde do Rio de Janeiro

Afrânio Coelho de Oliveira

Secretaria de Vigilância à Saúde/MS

$M$ aria de Fátima Souza M arinho

Secretaria de Atenção à Saúde/M S

Vânia Lucia Estrela

D epartamento de Atenção Básica/ MS

Andréa Leitão Ribeiro

$M$ arcela de Paula $M$ ateus

Instituto $\mathrm{N}$ acional de $\mathrm{C}$ âncer/M S

Alexandre D onato

Anke Bergmann

Carlos José Coelho Andrade

Carlos M anoel Araújo

César Augusto Lasmar Pereira

Claudia N aylor

Eliane M oscoso Braga Teixeira

Eliane Pinto

Elizete $M$ artins dos Santos

ElyeteC anella

Gilberto Amorim

Itamar Tavares de Souza

José Bines

$M$ argarida Tutungi

M aria Célia Resende

$M$ ariângela Freitas Lavor

Sergio $\mathrm{M}$ elo

Teresa Caldas Camargo

Área T écnica da Saúde da M ulher/M S

Ana M argareth Gomes Leite

Ivone Peixoto Gonçalves

M aria M agy

Rosilene Brito Silva
Verônica Reis

Sociedade Brasileira de M astologia

Antonio Figueira Filho

D iógenes Baségio

Ézio $\mathrm{N}$ ovais D ias (presidente)

José Antonio Ribeiro

Sergio Zerbini Borges

Sociedade Brasileira de Cuidados Paliativos

Ricardo Caponero

Sociedade Brasileira de Radiologia/Radioterapia

Eduardo $M$ artella

Colégio Brasileiro de Radiologia

$\mathrm{H}$ ilton Kock

Sociedade Brasileira de Patologia

H elenice G obbi

Luiz Antonio Rodrigues de Freitas

H ospital M arter Dei (Belo H orizonte/M G)

H enrique Salvador

H ospital Albert Einstein

Sergio Daniel Simon

$\mathrm{N}$ úcleo M ama do H ospital Moinhos do Vento/RS

M aíra Caleff

D epartamento de M edicina Preventiva/U SP

José Eluf $N$ eto

Federação Brasileira de G inecologia e 0 bstetrícia

Jurandir M oreira de Andrade

Universidade Federal de Campinas/U N IC AM P

Luiz Carlos Zeferino

Universidade Federal do Espírito Santo

$M$ aria H elena Costa Amorim

Universidade Federal do Rio de Janeiro

O Iga $\mathrm{H}$ elena Renelles

Universidade Federal do Paraná

Vinícius M ilani Budel

Escola Nacional de Saúde Publica/FIO CRUZ

Sergio Koifman

U N AM AM A - União Nacional Solidária no Combate

ao Câncer de M ama

M arilena Garcia

M arli S. M assaú

Rosina Tognetti

SO S/C O RPO Gênero e Cidadania

Solange Rocha

AD AM A Associação dos Amigos da M ama de $\mathrm{N}$ itéroi

Tereza Cipreste

REDEH Rede de D esenvolvimento H umano

Thais Corral

Associação Viva a Vida

Wilma Godoy Almeida 